\title{
El estado de derecho: ¿al servicio de los derechos fundamentales?
}

\author{
Gonzalo Candia Falcón*
}

\begin{abstract}
RESUMEN
Este trabajo busca demostrar que los desiderata del Estado propuestos por Lon Fuller promueven la protección de los derechos fundamentales. Esto en la medida que su cumplimiento necesariamente limita la capacidad de la autoridad -sea esta dictatorial, totalitaria o democrática-para infringir dichos derechos. En este contexto, el autor también plantea que los desiderata del estado de derecho no están exclusivamente al servicio de fines puramente formales, como asegurar la eficacia del sistema jurídico, sino que también ellos buscan permitir a las personas el ejercicio significativo de su libertad personal. Mediante aquello, el estado de derecho reconoce la dignidad de las personas, fundamento último de los derechos.
\end{abstract}

Estado de derecho - moralidad interna del derecho - derechos fundamentales

\section{The Rule of Law: At the Service of Fundamental Rights?}

\begin{abstract}
This article seeks to demonstrate that the desiderata of the rule of law proposed by Lon Fuller promote the protection of fundamental rights. This is because those standards, when sufficiently implemented by the ruler, necessarily restrict the ability of government-be it dictatorial, totalitarian or democratic-to infringe rights. In this context, the author also points out that the desiderata of the rule of law are not only at the service of formal purposes, such as to improve the efficacy of the legal system, but also they allow people to exercise their freedom meaningfully. In doing so, the rule of law recognizes the dignity of the person, which is the ultimate foundation of rights.
\end{abstract}

Rule of law - internal morality of the law - fundamental rights

* Abogado. Doctor en Derecho, Universidad de Georgetown. Profesor de Derecho Constitucional y Derecho Internacional de los Derechos Humanos, Facultad de Derecho, Pontificia Universidad Católica de Chile. Correo electrónico: gfcandia@uc.cl.

El autor agradece los útiles, precisos y detallados comentarios proveídos por los dos árbitros del presente artículo, los que permitieron su formulación definitiva. Del mismo modo, agradece los comentarios ofrecidos por el profesor Dr. Francisco Javier Urbina y el Dr. John Brungardt. Las traducciones son originales del autor.

Artículo recibido el 3.3.2016 y aceptado para su publicación el 10.7.2017. 


\section{INTRODUCCIÓN}

$\mathrm{L}$ a expresión estado de derecho es de uso corriente en la teoría política y jurídica contemporánea. Distintas significaciones han sido atribuidas a tal concepto por parte de la doctrina ${ }^{1}$. Sin embargo, una de las versiones paradigmáticas de estado de derecho es aquella desarrollada por Lon Fuller en The Morality of Law. Básicamente, esta noción vincula las exigencias del estado de derecho a la satisfacción de aquellos requerimientos formales sin los cuales un sistema jurídico no puede operar adecuadamente, así como la generalidad, la no retroactividad o la predictibilidad. Los mismos son denominados por Fuller como los desiderata del estado de derecho. Este trabajo buscará demostrar que la realización suficiente de esos desiderata por parte de un sistema jurídico no solo permite dotar de eficacia al ordenamiento, sino que también permite proteger derechos fundamentales.

Para testear la capacidad conceptual del estado de derecho para proteger derechos fundamentales se evaluará si la noción en cuestión permite ofrecer protección a los derechos en el contexto de un régimen cuyo poder se asienta en la infracción sistemática y permanente de los derechos fundamentales. En efecto, se argumentará que si el estado de derecho es capaz de ofrecer ciertas protecciones a los derechos fundamentales en un contexto como el descrito, con más razón podrá ofrecer esas mismas salvaguardas en un régimen en el que el respeto por los derechos fundamentales es criterio esencial de legitimidad política, como la democracia.

En relación con la estructura del artículo, este seguirá el siguiente orden. En primer lugar (i) se describirá el modelo de estado de derecho propuesto por Lon Fuller. Luego (ii) se procederá a analizar si dicho modelo tiene capacidad para proteger derechos fundamentales en el contexto de un régimen cuyo poder se funda en la violación permanente y sistemática de aquellos derechos. Este análisis concluirá que la sujeción instrumental de un gobernante injusto a criterios de estado de derecho permite proteger derechos fundamentales. Esto porque en la medida que esa sujeción sea efectiva, ella necesariamente limita la capacidad de maniobra de aquel gobernante para infringir derechos.

A partir de lo anterior, (iii) se afirmará que si los desiderata del estado de derecho tienen cierta capacidad para proteger derechos fundamentales en el contexto del ejercicio del poder por parte de un gobernante injusto, con más razón tendrán esa capacidad en el contexto de un régimen como el democrático, cuya legitimidad está fundada, precisamente, en el respeto por los derechos fundamentales. Para probar esa afirmación, (iv) se analizará jurisprudencia tanto del Tribunal Europeo de Derechos Humanos como de la Corte Interamericana de Derechos Humanos. Tras esto, se expondrá la conclusión.

${ }^{1}$ Tamanaha, B., On the rule of law. History, politics, theory, Cambridge University Press, Cambridge, 2004, p. 91. 


\section{El CONCEPTO DE ESTAdo DE DEREChO: LON Fuller}

Uno de los modelos de estado de derecho más difundidos en la doctrina es aquel presentado por Lon Fuller en su obra The Morality of Law. Para Fuller, la noción de estado de derecho está íntimamente asociada a su concepción del derecho. En este sentido, Fuller afirma que el derecho, en términos generales, debe ser concebido como la "empresa de sujetar la conducta humana al gobierno de las reglas" 2 . Ahora bien, la realización del derecho, en cuanto empresa, puede ser exitosa o bien culminar en un rotundo fracaso ${ }^{3}$. En ese contexto, Fuller planteó la historia de Rex, un rey imaginario que fracasa estrepitosamente en sus esfuerzos por generar normas que de forma efectiva sujetaran las conductas de sus gobernados conforme a ciertas reglas. A partir de las razones por las que Rex falló en su intento, Fuller enumera un conjunto de ocho patologías jurídicas ${ }^{4}$. Frente a esas ocho patologías, el autor en cuestión plantea la existencia de ocho virtudes esenciales que, conjuntamente consideradas, son condiciones de existencia de todo ordenamiento jurídico y representan la denominada "moralidad interna del derecho" 5 . Dichas virtudes representan en su conjunto las exigencias -los desiderata-del estado de derecho ${ }^{6}$. Los desiderata en cuestión son: (a) generalidad; (b) publicidad; (c) prospectividad; (d) claridad; (e) coherencia; ( $f$ ) posibilidad de ser cumplidas por sus destinatarios; ( g) estabilidad y (h) congruencia entre la norma preestablecida y su aplicación por parte de las autoridades ${ }^{7}$. Estas ocho características son representativas, de acuerdo a Fuller, "de los estándares de excelencia bajo los cuales toda legalidad debe ser evaluada" 8 . Ahora bien, el cumplimiento de estas exigencias por parte de los sistemas jurídicos no es, de acuerdo con Fuller, una cuestión puramente binaria, sino una que admite diversos grados de cumplimiento 9 . Así, un sistema jurídico es técnicamente más perfecto en la medida que responda de mejor manera a las exigencias contenidas en los desiderata; por el contrario, un sistema jurídico es técnicamente menos perfecto en la medida que responda de peor manera a las exigencias de aquellos.

En efecto, de acuerdo con Fuller, la satisfacción suficiente de los desiderata del estado de derecho por parte de los sistemas jurídicos permite que los mismos tengan la calidad técnica que requieren sus destinatarios para encontrarse en situación de obedecerlo. En ese sentido, un ordenamiento jurídico cuyo grado de cumplimiento de los desiderata sea tan bajo que el mismo sea incapaz de orientar la conducta de sus destinatarios "no resultaría ser un sistema jurídico deficiente, sino (...) algo que no puede ser llamado

\footnotetext{
${ }^{2}$ Fuller, L., The morality of law, Yale University Press, New Haven, 1969, p. 106.

${ }^{3}$ Ibid., pp. 117-118.

${ }^{4}$ Ibid., p. 39.

${ }^{5}$ Ibid., p. 42.

${ }^{6}$ Ibid., p. 214.

${ }^{7}$ Ibid., pp. 46-91.

${ }^{8}$ Ibid., p. 42.

${ }^{9}$ Ibid., p. 45.
} 
propiamente sistema jurídico" ${ }^{10}$. En ese sentido, los desiderata se asemejan a las reglas básicas de carpintería, cuyo cumplimiento permitiría al artesano dar vida a su obra ${ }^{11}$. Ello, evidentemente, sin perjuicio que ese mismo artesano pueda cumplir simultáneamente con otro tipo de reglas que perfeccionarían su obra desde otras perspectivas ${ }^{12}$. Esta comparación ha llevado a que autores como John Finnis asocien los desiderata planteados por Fuller con los requerimientos técnicos necesarios para que todo sistema jurídico opere "en forma"13.

Es evidente que los desiderata del estado de derecho planteados por Fuller tienen carácter esencialmente formal; esto es, los mismos buscan dotar a las reglas de una calidad técnica tal que permita que las mismas califiquen como derecho. Ello ocurrirá en la medida que esas reglas sean capaces de orientar la conducta de sus destinatarios, lo que, a su vez, permitirá su cumplimiento. Sin embargo, para Fuller, detrás del cumplimiento de las formalidades de los desiderata por parte de la autoridad que crea y administra el derecho, subyacen exigencias de naturaleza sustantiva. En efecto, Fuller califica el proceso de creación y aplicación del derecho como una empresa de colaboración entre el gobernante y los gobernados ${ }^{14}$. En ese contexto, la relación existente entre ambas partes es de reciprocidad ${ }^{15}$.

De acuerdo con esa relación de reciprocidad, ambas partes en la empresa común tienen obligaciones mutuas. Así, los destinatarios de las normas asumen la obligación moral de obedecer el derecho. De la misma forma, las autoridades tienen una obligación moral de generar derecho, esto es, un conjunto de normas que sean susceptibles de ser obedecidas en la medida que las mismas cumplan con los desiderata del estado de derecho ${ }^{16}$. Es el cumplimiento de esa obligación por parte de quienes crean y administran el derecho lo que justifica moralmente que su incumplimiento por parte de sus destinatarios pueda ser castigado por la autoridad ${ }^{17}$. Pero del mismo modo, el incumplimiento radical de los desiderata del estado de derecho por parte de quienes crean y administran el derecho priva de esa potestad sancionatoria a la autoridad. Esto en la medida que un derecho que no satisface criterios mínimos de generalidad, prospectividad, predictibilidad, claridad o coherencia simplemente no es, en la práctica, susceptible de ser obedecido por las personas ${ }^{18}$.

En conclusión, el modelo de estado de derecho planteado por Lon Fuller se funda en la existencia de ciertas exigencias formales -los desiderata- que todo ordenamiento jurídico debe satisfacer adecuadamente. La necesidad de ese cumplimiento suficiente

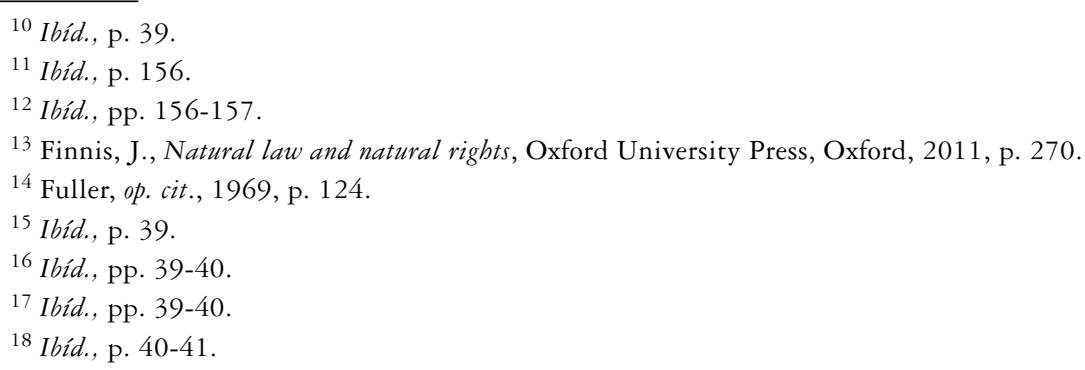


por parte de la autoridad que crea y administra el derecho se funda en razones tanto técnicas - un sistema jurídico que no lo haga impide a sus destinatarios obedecerlo- como morales -si la autoridad no obra de acuerdo con los desiderata, incumple sus obligaciones en el contexto de la empresa jurídica- .

\section{El MOdelo de ESTAdo DE DERECHO DE Fuller y LA PROTECCión DE LOS DERECHOS FUNDAMENTALES}

En la siguiente sección del presente trabajo se buscará responder esta pregunta: el modelo de estado de derecho planteado por Lon Fuller ¿tiene algún grado de capacidad para proteger los derechos fundamentales de las víctimas de un gobernante cuyo poder se asienta en la infracción sistemática y permanente de esos derechos? Si el modelo de estado de derecho propuesto por Fuller es capaz de ofrecer algún tipo de protección a los derechos en un régimen como el descrito, con más razón podrá hacerlo en un régimen como el democrático que, precisamente, funda su legitimidad en el respeto por aquellos derechos.

\section{Los desiderata del estado de derecho permiten proteger derechos fundamentales en el contexto de regímenes injustos}

Para determinar si el cumplimiento de las exigencias contenidas en los desiderata del estado de derecho por parte de un gobernante injusto permite proteger de alguna forma los derechos fundamentales de las víctimas de aquel, es necesario entender el contexto y las motivaciones por las que un régimen infractor de derechos fundamentales pudiese estar interesado en sujetarse a la disciplina impuesta por el estado de derecho.

En principio, para Fuller, la adhesión por parte de la autoridad que crea y administra el derecho a los desiderata se funda en la necesidad que tiene aquella de cumplir con su obligación moral en el contexto de la relación de reciprocidad que mantiene con los gobernados. Así, es posible entender que el cumplimiento de esa obligación moral no solo busca permitir el desarrollo de sistemas jurídicos eficaces, sino también afirmar valores sustantivos que subyacen a la noción misma de estado de derecho ${ }^{19}$. En efecto, el respeto por parte de la autoridad de los imperativos del estado de derecho no se justificaría únicamente en un criterio de eficacia legal, sino también en la necesidad de que los sistemas jurídicos reconozcan y promuevan la dignidad de la persona, fundada esta en el carácter libre y racional del individuo ${ }^{20}$.

En ese sentido, la finalidad de generar un conjunto de reglas jurídicas cuyas formas, contenidos y efectos sean capaces de orientar la actividad de las personas que viven en

19 Waldron, J., The rule of law and the measure of property, Cambridge University Press, Cambridge, 2012, p. 51.

${ }^{20}$ Fuller, op. cit., 1969 , pp. 167-168. 
sociedad implica asumir que los destinatarios de aquellas normas son seres racionales y libres, dotados de capacidad para deliberar y elegir entre diversas opciones ${ }^{21}$. Por tanto, la noción de estado de derecho en cuestión "no es neutral respecto del concepto de persona" 22 en la medida que la misma tácitamente reconoce a los destinatarios de las normas jurídicas como "agentes responsables, capaces de entender y seguir las reglas, y responder jurídicamente respecto de sus faltas" 23 .

Es, precisamente, esa racionalidad y libertad la que es servida por la autoridad que crea y administra el derecho al momento que esta satisface adecuadamente las exigencias de los desiderata del estado de derecho. Ahora bien, permitiendo el ejercicio significativo de esa libertad, los desiderata del estado de derecho reconocen implícitamente el valor de la dignidad de las personas. Ello porque el respeto por la dignidad humana exige que el derecho trate a las personas como individuos "capaces de planificar y ordenar su futuro" 24 en conformidad con sus propios proyectos de vida y de acuerdo con las exigencias del bien común de la comunidad política. En este escenario, la existencia de sistemas jurídicos que no sean lo suficientemente capaces de satisfacer en su conjunto los desiderata del estado de derecho representan "una afrenta a la dignidad de la persona en cuanto agente responsable" 25 .

Desde la perspectiva anteriormente descrita, un gobernante que funde su poder en la infracción sistemática y permanente de los derechos fundamentales carece de razones autosuficientes para sujetarse a los desiderata del estado de derecho ${ }^{26}$. En efecto, un gobernante que aterroriza a sus ciudadanos por medio de la violación sistemática de sus derechos fundamentales claramente es uno que no tiene, en la práctica, ningún tipo de interés en: (a) reconocer la dignidad de todas las personas sujetas a su poder -especialmente de sus opositores-, y (b) generar estructuras normativas que hagan posible el ejercicio significativo de la libertad de las personas a quienes tiraniza. Por tanto, este tipo de gobernante, desde una perspectiva conceptual, no tiene ninguna razón de tipo autosuficiente para justificar su adhesión al estado de derecho.

A pesar del análisis conceptual descrito, es posible apreciar que en la historia del siglo XX muchos gobernantes que fundaron su poder en la violación sistemática y permanente de los derechos humanos efectivamente trataron de exhibir ciertos grados de sujeción a las exigencias de los desiderata del estado de derecho. Este hecho permitiría afirmar que la noción de estado de derecho sería absolutamente irrelevante desde la perspectiva de la protección de los derechos. En efecto, si un gobernante que afianza su

${ }^{21}$ George, R., "Reason, freedom, and the rule of law: their significance in the natural law tradition", American Journal of Jurisprudence, Vol. 46, 2001, p. 255.

${ }^{22}$ Fuller, op. cit., 1969, p. 162.

23 Ibid., p. 162.

${ }^{24}$ Ibid., p. 221.

${ }^{25}$ Ibid., p. 162. Véase también: Rundle, K., Forms liberate. Reclaiming the jurisprudence of Lon L. Fuller, Hart Publishers, Oxford, 2013, pp. 97-101.

${ }^{26}$ Finnis, op. cit., pp. 273-274. Similar idea en: Douglas-Scott, S., Law after modernity, Hart Publishers, Oxford, 2013, p. 269. 
poder en la explotación sistemática de los derechos de los integrantes de la comunidad política no tiene problema alguno en sujetarse a las directrices del estado de derecho, entonces la noción en cuestión sería completamente incapaz de proveer algún tipo de protección a los mismos.

Sin embargo, un nuevo análisis conceptual puede ayudar a clarificar el contexto de la discusión. Si un gobernante injusto no tiene razones autosuficientes para someterse a la disciplina impuesta por el estado de derecho, entonces aquel solo tiene razones puramente instrumentales para hacerlo. Por tanto, la supuesta adhesión al estado de derecho manifestada por este tipo de gobernantes no está motivada en la necesidad de responder a la obligación moral derivada de la relación de reciprocidad mencionada por Fuller, sino en la necesidad de alcanzar algún tipo de propósito ulterior conveniente a los intereses del gobernante injusto.

Considérese algunas de esas razones instrumentales que podrían motivar a un gobernante injusto para someterse a las exigencias del estado de derecho: (a) el deseo del gobernante injusto de hacer más eficaz su maquinaria de dominación y (b) la necesidad del gobernante injusto de mejorar la percepción política de su régimen tanto dentro como fuera de su país. Se examinará a continuación si los desiderata del estado de derecho en estos contextos hipotéticos pueden ofrecer algún tipo de protección a los derechos fundamentales de las víctimas de un régimen injusto.

Primero, imagínese un tirano que decide aumentar los grados de precisión, claridad y determinación de su sistema jurídico con el propósito de hacer más eficaz su maquinaria represora. En un contexto como el descrito, parte de la doctrina cree que un modelo de estado de derecho como el descrito por Fuller en manos de un gobernante injusto podría garantizar solamente que "el transporte de la minoría perseguida al campo de concentración (...) sea sujeto al imperio de leyes debidamente promulgadas y escrupulosamente observadas ${ }^{27}$ ". Sin embargo, un análisis más profundo permite llegar a conclusiones opuestas. En efecto, incluso la sujeción superficial e interesada de un gobernante injusto a las exigencias del estado de derecho podría limitar la capacidad de maniobra del régimen en cuestión y, por medio de esa limitación, proteger derechos de las víctimas ${ }^{28}$.

Ello porque la formulación de toda regla jurídica en conformidad a las exigencias del estado de derecho supone que la misma sea redactada con un grado de determinación tal que permita a sus destinatarios distinguir los casos cubiertos por la regla de aquellos que no. Esto con un doble propósito. Primero, orientar adecuadamente la conducta de los destinatarios de la regla y permitir su cumplimiento; y, segundo, asegurar un

${ }^{27}$ Bingham, T., The rule of law, Penguin Books, Londres, 2011, p. 85.

${ }^{28}$ Finnis, op. cit., p. 274; George, op. cit., p. 253; Summers, R., "A formal theory of the rule of law", Ratio Iuris, Vol. $6 \mathrm{~N}^{\circ}$ 2, 1993, pp. 139. Célebre es, en este respecto, la referencia a E.P. Thompson, quien, adhiriendo a una concepción marxista de la historia, no dudó en afirmar que la noción de estado de derecho permitía limitar el poder político de la clase dominante en sus relaciones con los grupos sociales explotados. Ello le permitió afirmar que el estado de derecho representaba "un bien humano absoluto" ("unqualified human good”). Thompson, E.P., Whigs and hunters, Penguin Books, Londres, 1977, p. 266. 
espacio en el que las personas puedan interactuar libremente de acuerdo con sus propios planes $^{29}$. En este contexto, el gobernante injusto solo podría castigar el incumplimiento de la regla en ciertos casos, pero no en otros. De la misma forma, el gobernante injusto estaría obligado a respetar las decisiones adoptadas libremente por las personas en la medida que las mismas no se opusieran al texto de la regla en cuestión. De esa forma, su capacidad de ejercer coerción en conformidad al derecho se ve reducida y su capacidad de infringir derechos fundamentales disminuida ${ }^{30}$. Es por esa razón que lo propio de todo régimen que infringe sistemáticamente derechos fundamentales sea la producción de un derecho deficiente en cuanto su determinación ${ }^{31}$. Ello porque un derecho de esa naturaleza permite, precisamente, a los funcionarios de gobierno infringir los derechos de las personas de forma mucho más discrecional y, de esa forma, generar mayores espacios para la arbitrariedad del tirano en el poder ${ }^{32}$.

En ese sentido, considérese el siguiente caso. Durante los primeros años del régimen de Mussolini en Italia, en la década de 1920 y 1930, algunos jueces italianos buscaron desarrollar estrategias que, sin dejar de aplicar la nueva legislación fascista, permitieran limitar lo más posible el alcance de la misma cuando ella conllevara la infracción de derechos. Para ello, esos jueces decidieron servirse del desideratum de congruencia propio del estado de derecho: esto es, que la administración del derecho por parte de cualquier autoridad debe sujetarse al tenor del mismo. De esta forma, aquellos jueces declararon como ilegales toda actuación de autoridades fascistas que no fuese estrictamente conforme al texto de la legislación ${ }^{33}$. Para satisfacer el criterio, estos jueces rechazaron el uso de mecanismos de interpretación finalista de la legislación, los que habrían permitido extender la aplicación de las reglas a muchos más casos que aquellos a los que estrictamente debía aplicarse ${ }^{34}$. De esa forma, la administración del derecho en conformidad con los desiderata debilitó, en este tipo de casos, la capacidad del régimen para ofender la dignidad de las personas al menos durante sus primeros años de existencia.

Segundo, imagínese que un gobernante injusto afirme su intención de someter la producción y administración de su sistema jurídico a las exigencias del estado de

${ }^{29}$ Simmonds, N., Law as a moral idea, Oxford University Press, Oxford, 2007, pp. 141-143.

${ }^{30}$ Simmonds, N., "Straightforwardly false: the collapse of Kramer's positivism", The Cambridge Law Journal, Vol. $63 \mathrm{~N}^{\circ} 1,2004$, p. 101.

${ }^{31}$ E.g: considérese, por ejemplo, el artículo 16 del Código Penal soviético de 1936, norma que permitía a los tribunales de justicia del régimen estalinista castigar criminalmente a todos aquellos ciudadanos culpables de incurrir en un acto "socialmente peligroso". Obviamente, ni el código penal ni la legislación general especificaban qué tipo de actos debían ser considerados por los jueces como "socialmente peligrosos". Véase: Grzybowski, K., "Main trends in the soviet reform of criminal law", The American University Law Review, Vol. $9 \mathrm{~N}^{\circ}$ 2, 1960, p. 97.

32 Summers, op. cit., p. 139.

${ }^{33}$ Calabresi, G., "Two functions of formalism: in memory of Guido Tedeschi”, The University of Chicago Law Review, Vol. 67, 2000, p. 482.

${ }^{34}$ Esta fue la tendencia interpretativa que, precisamente, siguieron los jueces alemanes al momento de aplicar la legislación racial nacionalsocialista. Véase: Muller, I., Hitler's justice: the courts of the Third Reich, Harvard University Press, Cambridge Massachusetts, 1991. 
derecho con la finalidad de elevar los grados de legitimidad política del régimen tanto al interior como hacia el exterior del país. En este escenario, algunos podrían afirmar que el estado de derecho efectivamente representa una amenaza para la protección de los derechos fundamentales. Ello porque esa adhesión superficial e interesada al estado de derecho por parte del gobernante injusto permitiría que este pudiese aumentar su validación nacional e internacional y, desde esa perspectiva, prolongar la existencia del régimen. Esto permitiría, a la larga, proyectar la infracción de derechos fundamentales en el tiempo.

Sin embargo, ello no debe ocurrir necesariamente así. En efecto, el compromiso superficial adoptado por aquel gobernante injusto con las exigencias del estado de derecho podría ser utilizado por sus víctimas para debilitarlo políticamente. En el escenario descrito, esas víctimas dispondrían de criterios de legitimidad aceptados por el propio gobernante injusto para juzgar las actuaciones del régimen tanto dentro como fuera del país. En la medida que este tirano se somete al estado de derecho únicamente de forma superficial -y entendiendo que un tirano no busca limitar, sino incrementar su poder cada día- es muy probable que surjan rápidamente contradicciones entre la voluntad manifestada por el gobernante injusto y sus actuaciones concretas. El juzgamiento de este tipo de acciones a la luz del estado de derecho por parte de las víctimas podría dar origen a ciertas críticas en contra del régimen en razón del incumplimiento de sus propias promesas ${ }^{35}$. Esas críticas tienen suficiente potencial para horadar la legitimidad del régimen y, por esta vía, debilitar su posición política ${ }^{36}$. En este contexto, es posible imaginar que ese gobernante injusto tratará de acallar las críticas nacionales e internacionales no solo mediante la violencia, sino también tratando de ajustar sus actuaciones a criterios de estado de derecho que vuelvan a legitimarlo. En la medida que ello ocurra se producirá el fenómeno descrito anteriormente: la producción y administración del derecho de acuerdo con criterios de estado de derecho por parte del gobernante injusto disminuirá su capacidad para infringir derechos fundamentales.

Considérese, por ejemplo, el caso sudafricano. Durante el régimen del apartheid, el gobierno enfatizaba permanentemente que su sistema jurídico, pese a fundarse en la discriminación racial, se ajustaba perfectamente a las exigencias del estado de derecho. Ello únicamente con el propósito de lograr el reconocimiento de la legitimidad de un sistema rechazado por la mayoría de la población sudafricana y de la comunidad internacional. Dicha adhesión superficial al estado de derecho por parte de las autoridades de Sudáfrica permitió que la oposición pudiese debilitar políticamente al régimen. Ello a partir de las críticas dirigidas por esa oposición hacia la legislación del apartheid que no se ajustaba a las exigencias mínimas del estado de derecho. Por otro lado, muchos de los jueces sudafricanos hicieron efectivas las limitaciones que la legislación sudafricana

35 Peerenbom, R., "Human rights and rule of law: what's the relationship?", Georgetown Journal of International Law, Vol. 36, 2004-2005, p. 945.

36 Waldron, J., "The rule of law in contemporary liberal theory", Ratio Iuris, Vol. $2 \mathrm{~N}^{\circ} 1,1989$, p. 93-94. Similar idea en: Summers, op. cit., 1993, p. 139. 
imponía a las autoridades refiriendo la necesidad de preservar el estado de derecho ${ }^{37}$. Esto muestra que la adhesión superficial de un régimen infractor de derechos a las exigencias del estado de derecho debilita la capacidad de ese gobierno para infringir derechos.

En conclusión: cuando un gobernante injusto decide sujetarse -aunque sea superficialmente y por razones espurias- a las exigencias del estado de derecho, se debilita su capacidad de maniobra y con ello se desgasta su poder para infringir derechos de las personas. Por esa vía el estado de derecho presta un servicio a la protección de los derechos. Desde esta perspectiva, un régimen injusto parece no tener buenas razones para sujetarse a la disciplina impuesta por el estado de derecho ${ }^{38}$.

\section{Los desiderata del estado de derecho permiten proteger derechos fundamentales en el contexto de regímenes justos}

En el apartado anterior se demostró que la sujeción, incluso superficial e interesada, por parte de un gobernante injusto a los desiderata del estado de derecho permite generar ciertas protecciones para los derechos fundamentales de las personas. Ahora bien, si los desiderata del estado de derecho prestan ese servicio a los derechos fundamentales en el contexto de regímenes cuyo propósito es, precisamente, infringirlos, con mucha mayor razón podrán realizar el mismo servicio en el contexto de regímenes como el democrático, cuya legitimidad política está fundada en la necesidad que la autoridad respete los derechos fundamentales de los ciudadanos.

En ese sentido, las instituciones de un régimen democrático tienen la obligación moral de producir y administrar el derecho en conformidad a las exigencias del estado de derecho. Dicha obligación moral se origina en el hecho que la legitimidad misma de todo gobierno democrático se funda no solo en su origen electivo sino también en el respeto que este promueve de la dignidad y de la libertad de las personas. En consecuencia, el caso focal $^{39}$ de una legislatura democrática buscará especificar el contenido de los derechos por medio de una legislación formulada en términos tales que la misma tenga capacidad suficiente para orientar la conducta de sus destinatarios, reconociendo con ello la dignidad de los mismos. Desde esa perspectiva, los desiderata del estado de derecho representan "las leyes implícitas del proceso legislativo" ${ }^{40}$, las que establecen límites específicos a toda autoridad legisladora dentro de un estado democrático ${ }^{41}$.

${ }^{37}$ Abel, R., "Legality without a Constitution: South Africa in the 1980s", en Dyzenhaus, D. (editor), Recrafting the rule of law, Hart Publishers, Oxford, 1999, pp. 64-65.

${ }^{38}$ Simmonds, op. cit., 2004, pp. 101-102.

39 El significado focal de algo representa el paradigma central de la realidad que se describe por medio de un término, en contraposición a los casos periféricos del mismo. Estos últimos comparten parte del significado focal, pero se diferencian de este debido a la presencia o ausencia de ciertos factores que lo distancian de aquel. Véase: Finnis, op. cit., pp. 9-11.

${ }^{40}$ Fuller, L., "The implicit laws of lawmaking”, en Winston, K. (edit.), The principles of social order. Selected essays of Lon L. Fuller, Hart Publishers, Oxford, 2001, p. 178.

${ }^{41}$ Ibid., p. 178. 
Así como se refirió, la principal responsabilidad del legislador democrático en relación con los derechos fundamentales es dictar leyes que permitan hacer efectivo el ejercicio de los mismos ${ }^{42}$. Esto es un deber que incluso está reconocido en los tratados internacionales de derechos humanos ${ }^{43}$. Ahora bien, el cumplimiento de este deber exige que la legislación dictada tenga la calidad técnica necesaria para coordinar el ejercicio de un conjunto de derechos y libertades reconocidos tanto por las constituciones nacionales como por los tratados internacionales de derechos humanos. Para cumplir con esta finalidad coordinadora, la legislación deberá especificar con propiedad los tipos de conductas que están permitidos o prohibidos en relación con el ejercicio de esos derechos y libertades. Esto, a su vez, implica fijar límites al ejercicio de esos derechos y establecer con claridad "las responsabilidades y sanciones que fueren necesarias para obtener tal propósito" ${ }^{44}$. Es este proceso de especificación el que permitirá, precisamente, armonizar el ejercicio de los distintos derechos con "las justas exigencias del bien común, en una sociedad democrática”, según indica el artículo 32.2 de la Convención Americana de Derechos Humanos.

En este proceso de especificación de derechos fundamentales, el legislador democrático tiene la obligación de cuidar que el esquema normativo que busca posibilitar el ejercicio de los derechos sea formulado de acuerdo con las exigencias de los desiderata del estado de derecho. Ello en el entendido que en todo régimen democrático sano existe un compromiso de fondo de este con el concepto de persona que subyace al ideal de estado de derecho planteado por Fuller.

En la medida que la legislación de derechos fundamentales satisfaga suficientemente los desiderata del estado de derecho, aquella permitirá un ejercicio de los derechos y libertades más pleno. Ello por dos razones. Primero, una legislación de esta naturaleza será capaz de orientar adecuadamente a sus destinatarios en relación con el contenido y alcance del ejercicio de sus derechos, por lo que aquellos podrán ejercerlos con mayor propiedad. Segundo, porque solo una legislación de derechos fundamentales dictada en conformidad con las exigencias del estado de derecho está capacitada para limitar de forma efectiva la discrecionalidad de la autoridad llamada a aplicar esa legislación.

Por el contrario, una legislación de derechos fundamentales que fuese deficiente en su formulación técnica - por no ajustarse suficientemente a las exigencias del estado de derecho- no podrá orientar adecuadamente a las personas en relación con la forma cómo deben ejercer sus derechos y tampoco permitirá establecer restricciones efectivas a la discrecionalidad de la autoridad llamada a aplicar esa legislación. Esto último es sumamente grave desde la perspectiva de derechos fundamentales. En efecto, una legislación acerca de estos derechos que carezca de suficiente precisión, determinación, certidumbre o previsibilidad no será capaz de erigirse como límite a la discrecionalidad

42 Webber, G., The negotiable constitution. On the limitations of rights, Cambridge University Press, Cambridge, 2012, pp. 135-138.

43 Véase en este respecto los artículos $1^{\circ}$ del Convenio Europeo de Derechos Humanos y los artículos $1^{\circ}$ y $2^{\circ}$ de la Convención Americana sobre Derechos Humanos.

${ }^{44}$ CIDH. Caso Tristán Donoso v. Panamá, párr. 112. 
de la autoridad ejecutiva o judicial llamada a aplicarla. En efecto, el alto grado de indeterminación y vaguedad de la legislación en cuestión impediría que, en la práctica, la autoridad referida dispusiese de un criterio cierto que restrinja su discrecionalidad. Consecuentemente, esa autoridad podría tomar decisiones en torno a los derechos fundamentales de las personas sin disponer de un criterio predeterminado que permitiese orientar y limitar su acción. A partir de lo anterior, es posible comprender que una legislación de esa naturaleza disminuiría de forma relevante la capacidad que tienen los derechos fundamentales para limitar a la autoridad política, con el consiguiente riesgo para el ejercicio de los propios derechos ${ }^{45}$.

Lo anterior explica por qué la conexión entre estado de derecho y derechos fundamentales sea reconocida incluso en el ámbito del derechos internacional de los derechos humanos. En efecto, el Tribunal Europeo de Derechos Humanos ha señalado que el legislador democrático no cumple su obligación internacional de implementar los derechos reconocidos en el Convenio Europeo por el solo hecho de dictar la legislación correspondiente. Por el contrario, esa obligación se vería satisfecha únicamente cuando esa legislación responda a ciertos estándares de calidad técnica, como la suficiente determinación de las normas ${ }^{46}$. Así, por ejemplo, tratándose de legislación que autoriza a la autoridad para restringir la libertad de las personas, aquella deberá estar "formulada con la precisión suficiente para permitir a los peticionarios anticipar o prever las consecuencias de sus acciones" ${ }^{2}$.

También la Corte Interamericana de Derechos Humanos ha asumido la doctrina en cuestión. En efecto, la Corte ha afirmado que toda legislación nacional que regule el ejercicio de derechos fundamentales no es compatible con las exigencias de la Convención Americana por el solo hecho de establecer en qué casos la autoridad tiene facultades para restringirlos. En efecto, esa compatibilidad también dependerá de la calidad técnica de aquella legislación, la que deberá ser formulada en términos tales que sea capaz de producir grados mínimos de previsibilidad en sus destinatarios ${ }^{48}$.

Ahora bien, si las jurisdicciones de derechos humanos señaladas han enfatizado la conexión entre los desiderata del estado de derecho y los derechos fundamentales no es solamente porque ellas se sientan animadas a hacerlo en razón de cuestiones puramente técnicas, e.g: la necesidad de promover ordenamientos jurídicos eficaces. Por el contrario, ese interés se debe a que los tribunales en cuestión también entienden que la infracción por parte del legislador democrático de los desiderata del estado de derecho puede tener como consecuencia directa la violación de derechos fundamentales por parte de las autoridades nacionales. Jurisprudencia tanto del Tribunal Europeo de Derechos Humanos como de la Corte Interamericana de Derechos Humanos dan cuenta de aquello.

\footnotetext{
${ }^{45}$ En relación con la importancia de los derechos como límites, véase: Webber, op. cit., 2012, pp. 123-133.

46 TEDH. Sunday Times $v$. Reino Unido, párr. 49.

47 TEDH. Steel y otros $v$. Reino Unido, párr. 75.

${ }^{48} \mathrm{CIDH}$. Caso Torres Millacura y otros v. Argentina, párr. 79-80.
} 
2.1. Estado de derecho y derechos fundamentales: casos en los que la infracción de uno conduce la violación de los otros

Los tribunales regionales de derechos humanos, como el europeo y el interamericano, han constantemente afirmado que la infracción de los desiderata del estado de derecho por parte del legislador democrático permite la violación de derechos fundamentales por las autoridades nacionales.

Invocando la violación del derecho a la privacidad, el Tribunal Europeo de Derechos Humanos condenó al estado en el caso Huvig vs. Francia (1990). El tenor del código de procedimiento penal francés vigente al tiempo en que ocurrieron los hechos del caso era muy poco claro al momento de definir si un juez instructor tenía atribuciones suficientes para interceptar conversaciones telefónicas en el contexto de una investigación criminal $^{49}$. De acuerdo con el Tribunal Europeo, esa falta de claridad no se condecía con los desiderata de claridad y congruencia. Ello porque la legislación en cuestión "no especificaba con claridad razonable los límites y las formas por medio de las cuales las autoridades podían ejercer [la] atribución discrecional [de intervenir teléfonos]" 50 . Esto, en la práctica, significaba privar a las personas de las "protecciones mínimas a las que debieron acceder bajo un estado de derecho en una sociedad democrática" 51 . Dicha infracción de las exigencias del estado de derecho permitió, a su vez, que un juez instructor en el contexto de una investigación por delitos tributarios violara el derecho a la privacidad del denunciante por medio de la intervención de sus conversaciones telefónicas, razón por la que el Tribunal condenó al Estado francés ${ }^{52}$.

Similar razonamiento siguió el Tribunal Europeo en Herczegfalvy vs. Austria (1992). En este caso, el Tribunal afirmó que ciertas disposiciones de la ley de hospitales austríaca no satisfacían las exigencias de congruencia del estado de derecho. Ello porque las normas en cuestión estaban redactadas de una forma tal que no eran capaces de generar un criterio predeterminado que permitiera delimitar las facultades discrecionales de la autoridad administrativa en relación con el manejo de la correspondencia recibida por quienes se encontrasen internados forzosamente en hospitales psiquiátricos ${ }^{53}$. La ausencia de determinación y claridad en torno a este punto llevó a afirmar al Tribunal que "las disposiciones cuestionadas no ofrecían el mínimo nivel de protección en contra de la arbitrariedad requerido por el estado de derecho" 54 . Ahora bien, la infracción al estado de derecho descrita permitió, en los hechos, que la autoridad de un hospital psiquiátrico negase permanentemente al internado denunciante el acceso a la correspondencia recibida desde el mundo exterior. En términos del Tribunal Europeo, fue la baja calidad técnica del derecho doméstico aplicable la que "expuso al internado denunciante a la

\footnotetext{
49 TEDH. Caso Huvig v. Francia, párr. 13-14.

${ }^{50}$ Ibid., párr. 35.

51 Ibid., párr. 35.

52 Ibid., párr. 35.

53 TEDH. Herczegfalvy v. Austria, párr. 91.

${ }^{54}$ Ibid., párr. 91.
} 
mera voluntad de las autoridades médicas" 55 . Todo ello permitió al Tribunal condenar al Estado austríaco por la infracción del derecho a la privacidad del denunciante.

En conformidad con el mismo patrón, el Tribunal Europeo condenó al Estado en Maestri vs. Italia (2004). De acuerdo con el Tribunal, el artículo 18 del Real Decreto Legislativo $\mathrm{N}^{\circ} 511$ de 1946 representaba, en conjunto con su normativa legal complementaria, una infracción del desideratum de predictibilidad demandado por el estado de derecho. La norma referida señalaba que todo juez "que no cumpliese sus obligaciones o bien se comportara (...) en una forma que le hiciera indigno de la (...) consideración que debe disfrutar" debía ser castigado disciplinariamente. En el caso concreto, la autoridad judicial invocó este precepto legal junto con otra normativa complementaria para sancionar a un juez en razón de su pertenencia a una lógica masónica. El Tribunal Europeo afirmó que el conjunto del derecho aplicado para sancionar al denunciante se encontraba formulado en términos tales que "el mismo no contenía información suficiente para satisfacer las exigencias de predictibilidad" 56 del estado de derecho. Por tanto, la normativa "no era lo suficientemente clara para permitir al denunciante (...) comprender (...) que su pertenencia a una logia masónica podía significar la aplicación de sanciones administrativas en su contra" ${ }^{\text {7 }}$. De acuerdo con el Tribunal, fue esa infracción del estado de derecho por parte del legislador la que permitió que las autoridades judiciales del Estado italiano violaran la libertad de conciencia del denunciante.

Siguiendo en la misma línea, el Tribunal Europeo condenó al Estado en el caso Gillan y Quinton vs. Reino Unido (2010). En su sentencia, el Tribunal afirmó que ciertas disposiciones de la legislación antiterrorista británica no se condecían con la idea de congruencia propia del estado de derecho. Ello en la medida que el derecho aplicable estaba redactado en términos tales que era incapaz de generar un criterio predeterminado susceptible de ser aplicado por las autoridades correspondientes. A este respecto, los preceptos legales en cuestión permitían a la policía detener a cualquier persona para efectos de realizar un control de identidad "si se considera[ba] conveniente para la prevención de actos de terrorismo" 58 . El Tribunal Europeo afirmó que dicha formulación legal otorgaba un poder de discrecionalidad absoluto al funcionario policial en relación con el ejercicio de la libertad personal, sin sujetarlo a límites preestablecidos 59 . Ahora bien, esta infracción del estado de derecho por parte del legislador democrático británico permitió la consiguiente violación de la libertad personal de los dos denunciantes del presente caso, quienes fueron detenidos y registrados arbitrariamente por la policía de Londres en 2003, razón por la que el Tribunal finalmente condenó a Reino Unido.

Similar patrón de casos se han dado en la jurisprudencia de la Corte Interamericana de Derechos Humanos. En este sentido, una de las sentencias más representativas es Caso Baena Ricardo y otros vs. Panamá (2001). En ella, la Corte Interamericana entendió que la

\footnotetext{
${ }^{55}$ Ibid., párr. 91.

56 TEDH. Maestri v. Italia, párr. 37.

${ }^{57}$ Ibid., párr. 41.

58 TEDH. Gillan y Quinton v. Reino Unido, párr. 29.

${ }^{59}$ Ibíd., párr. 83.
} 
Ley $\mathrm{N}^{\circ} 25$ de 1991, dictada por el Congreso panameño en respuesta a una huelga general de funcionarios públicos realizada el 5 de diciembre de 1990, era incompatible con el desideratum de no retroactividad requerido por el estado de derecho. El artículo $6^{\circ}$ de la mencionada ley facultó a la autoridad ejecutiva para destituir retroactivamente a todos los funcionarios que hubieran participado de aquella huelga. De acuerdo con la Corte Interamericana, la norma en cuestión no era admisible en el contexto de "un estado de derecho [en el que] los principios de legalidad e irretroactividad presiden la actuación de todos los órganos del Estado" 60 . En ese escenario, la Corte afirmó que la formulación del artículo $6^{\circ}$ de la Ley $\mathrm{N}^{\circ} 25$ de 1991 no permitió que sus destinatarios "[hubiesen podido] orientar su comportamiento conforme a un orden jurídico vigente y cierto" 61 al tiempo de la ocurrencia de los hechos. En efecto, de acuerdo con la Corte, esa infracción al estado de derecho permitió la violación, por parte del ejecutivo panameño, de los derechos de libertad de asociación y debido proceso de los 270 trabajadores cesados.

Similar vínculo entre estado de derecho y protección de derechos se aprecia en la sentencia Fermín Ramírez vs. Guatemala (2005). En este caso, la Corte Interamericana afirmó que el artículo 132 del código penal guatemalteco no se condecía con las exigencias del desideratum de congruencia. Esto en la medida que la norma legal en cuestión estaba redactada en términos tales que era incapaz de ofrecer al juez penal un criterio predeterminado para decidir en qué casos aplicar la pena de muerte tratándose de condenados por homicidio calificado. En este sentido, la disposición establecía que ese tipo de delitos debía castigarse con una pena de presidio entre 25 y 50 años. Sin embargo, en caso que el condenado "revelare una mayor particular peligrosidad" el juez podía discrecionalmente decidir si reemplazar la pena de presidio por la aplicación de la pena de muerte ${ }^{62}$. Las deficiencias técnicas de la redacción del artículo 132 del código penal permitieron que los tribunales de Guatemala impusieran la pena de muerte a Fermín Ramírez, quien había sido condenado previamente por la comisión del delito de homicidio calificado. La Corte Interamericana, en este caso, condenó al Estado por la violación del derecho a la integridad física y al debido proceso de la víctima.

En Kimel vs. Argentina (2007), la Corte Interamericana, tras analizar la regulación penal argentina en torno a los delitos de injuria y calumnia, concluyó que la misma era altamente deficiente desde la perspectiva de los desiderata de claridad y coherencia del estado de derecho. Ello en la medida que los artículos 109 y 110 del código penal argentino no respondían adecuadamente al estándar básico de precisión y univocidad exigible a toda norma jurídica de carácter penal ${ }^{63}$. Esto, en la práctica, permitió que el denunciante -el escritor Eduardo Kimel- fuera condenado por el tribunal de primera instancia, de acuerdo con el artículo 110 del código penal, por el delito de injurias y, tras la revocación de la condena por injurias en el tribunal de apelación, fuera condenado

\footnotetext{
${ }^{60}$ CIDH. Caso Baena Ricardo y otros v. Panamá, párr. 107.

${ }^{61}$ Ibid., párr. 106.

${ }^{62}$ CIDH. Caso Fermín Ramírez v. Guatemala, párr. 94.

${ }^{63}$ CIDH. Kimel v. Argentina, párr. 63.
} 
esta vez por la comisión del delito de calumnias por la Corte Suprema. De acuerdo con la Corte Interamericana, la infracción de los desiderata de coherencia, claridad y determinación - producida por una "deficiente regulación penal de esta materia" 64 - permitió, en la práctica, que los tribunales argentinos violaran el derecho a la libertad de expresión del denunciante en este caso.

Finalmente, la Corte Interamericana afirmó en Caso Torres Millacura y otros vs. Argentina (2011) que el artículo 10 letra b) de la Ley $N^{\circ} 815$ de la provincia de Chubut era incompatible con los desiderata de predictibilidad y de congruencia del estado de derecho. Una vez más, el precepto legal era incapaz de originar un criterio predeterminado concreto que permitiese a la autoridad correspondiente someterse al mismo. Ello en cuanto la ley autorizaba a la policía provincial para detener una persona con el propósito de practicar un control de identidad cuando fuese "necesario conocer sus antecedentes en circunstancias que lo justifiquen”. De acuerdo con la Corte, la disposición en comento carecía de la especificidad suficiente para que las personas pudiesen prever debidamente las consecuencias de sus actuaciones ${ }^{65}$. Por otro lado, la norma en cuestión "no precisaba concretamente los supuestos por los cuales los policías podían demorar [detener] a una persona con la finalidad de identificarla" ${ }^{66}$. La Corte reconoció que esta infracción de las exigencias del estado de derecho permitió, en la práctica, que la policía de Chubut violara el derecho a la vida y la libertad personal del joven chileno Iván Torres Millacura, quien el 26 de septiembre de 2003 fue detenido en conformidad a lo dispuesto en la ley mencionada, ejecutado extrajudicialmente y hecho desaparecer.

En conclusión, la jurisprudencia tanto del Tribunal Europeo de Derechos Humanos como de la Corte Interamericana de Derechos Humanos considera que la infracción de los desiderata del estado de derecho por parte del legislador democrático facilita la violación de derechos fundamentales por la autoridad llamada a administrar el derecho. A partir de lo anterior es posible comprender que la producción de un derecho conforme a ciertos criterios de excelencia técnica por parte del legislador democrático no solo permite dotar de eficacia al ordenamiento, sino que también permite proteger derechos fundamentales en el contexto de un régimen de gobierno democrático. Esta circunstancia permite afirmar con Fuller que en la mayoría de los casos existe una conexión más que una contradicción entre legalidad y justicia ${ }^{67}$.

\section{EL RÉGIMEN DEMOCRÁTICO Y LOS LÍMITES DEL ESTADO DE DERECHO}

No obstante lo señalado en el acápite anterior, la tesis de que los desiderata del estado de derecho coadyuvan a la protección de los derechos fundamentales en el contexto de un

\footnotetext{
${ }^{64}$ Ibid., párr. 66.

${ }^{65}$ CIDH. Caso Torres Millacura y otros v. Argentina, párr. 79-80.

${ }^{66}$ Ibid., párr. 79.

${ }^{67}$ Fuller, op. cit., 1969, p. 157.
} 
régimen democrático debe confrontar el hecho de que, en muchos casos, los legisladores podrían utilizar -al igual que los gobernantes injustos descritos en la primera parte de este trabajo- las exigencias del estado de derecho para realizar fines que no se condicen, precisamente, con la salvaguarda de los derechos.

En relación con este punto, conviene recordar que el mismo Fuller señalaba que "la aceptación y el cumplimiento de las exigencias propias de la moralidad interna del derecho son una condición necesaria, pero no suficiente para la realización del ideal de justicia" 68 . Sin embargo, ello no significa que el estado de derecho en democracia sea irrelevante para la protección de los derechos fundamentales. Por el contrario, el servicio que el estado de derecho presta a los derechos y libertades en el contexto de un régimen democrático sigue siendo significativo, aun cuando la legislatura busque instrumentalizarlo indebidamente.

En efecto, aun en ese escenario, las exigencias del estado de derecho continúan prestando un importante servicio a los derechos fundamentales. En efecto, la sujeción de la legislatura injusta a los desiderata del estado de derecho permite: (a) limitar su discrecionalidad y (b) restringir el impacto del derecho promulgado. Asimismo, las exigencias del estado de derecho en el contexto señalado sirven de estándares que permiten juzgar la acción de los legisladores en el foro público. En ese sentido, los ciudadanos podrían invocar la legitimidad de los desiderata para señalar la injusticia y promover el cambio de la legislación. Este tipo de acción está llamado a tener un alto grado de efectividad en el contexto de un régimen democrático, en el que la libertad de expresión, la transparencia y la deliberación son pilares esenciales del sistema. Adicionalmente, los ciudadanos podrían combatir al legislador injusto por medio del voto en elecciones periódicas, lo que permitiría hacer efectiva la responsabilidad política de aquel. Finalmente, en la gran mayoría de los sistemas constitucionales democráticos existen instituciones independientes, como los tribunales de justicia, que dificultan la acción del legislador injusto.

A lo señalado podría reiterarse que, a pesar de los argumentos ofrecidos, es todavía posible imaginar casos en los que una legislatura democrática manipule las exigencias del estado de derecho para infringir derechos fundamentales sin que ello genere mayor resistencia ni de la población, ni del resto de las instituciones del Estado. Esto permitiría finalmente demostrar que el estado de derecho carece de relevancia para proteger derechos en democracia.

A este respecto es posible señalar que la realización de los efectos virtuosos del estado de derecho al interior de cualquier comunidad política requiere de la existencia de un contexto político y normativo adecuado que permita su operatividad ${ }^{69}$. Ello porque el estado de derecho no es un concepto que opere con independencia de la cultura y de las instituciones de una comunidad política. En efecto, el estado de derecho tendrá mucha más capacidad para proteger derechos fundamentales en democracia cuando este opere

${ }^{68}$ Ibid., p. 168.

${ }^{69} \mathrm{Raz}, \mathrm{J}$., "The politics of the rule of law”, en Raz, J. (autor), Ethics in the public domain, Oxford, Oxford University Press, 1995, pp. 375-378. Similar idea en: Peerenboom, op. cit., p. 945. 
en el contexto de una sociedad políticamente madura y dotada de instituciones independientes. Por el contrario, el estado de derecho tendrá menor capacidad para proteger derechos fundamentales en democracia cuando la sociedad en cuestión sea políticamente inmadura y sus instituciones no sean independientes o bien, siéndolo, carezcan de los recursos humanos y materiales necesarios para actuar adecuadamente.

En este último escenario, la noción de estado de derecho propuesta por Fuller será claramente menos eficiente para custodiar derechos en democracia. Sin embargo, esto no es consecuencia del supuesto carácter formal de la noción de estado de derecho propuesta por Fuller. Por el contrario, aquel menor grado de eficiencia es consecuencia de las profundas debilidades que un sistema democrático puede experimentar en el contexto de una sociedad políticamente inmadura y altamente manipulable, carente de instituciones independientes. Son estas circunstancias -y no la noción de estado de derecho utilizada en este trabajo- las que facilitarían o bien permitirían la violación de derechos por parte de una legislatura injusta en aquella sociedad.

De hecho, aun en un contexto tan adverso para los derechos fundamentales como el descrito, el estado de derecho todavía puede prestar un servicio a la protección de derechos fundamentales en democracia. Si efectivamente la legislatura injusta busca infringir derechos por medio de la dictación de normas conformes a las exigencias del estado de derecho, dichas exigencias limitarán la aplicación del criterio injusto. Por otro lado, los estándares del estado de derecho ofrecen criterios que las víctimas podrían utilizar para, por ejemplo, recurrir a tribunales internacionales de derechos humanos con el propósito de obtener la condena del Estado y visibilizar la problemática del país ante la comunidad internacional ${ }^{70}$. A partir de lo anterior, pareciera que la legislatura injusta en cuestión no debiese sentir un particular interés en sujetarse plenamente a las exigencias del estado de derecho para infringir derechos fundamentales.

\section{UNA PROPUESTA DE SOLUCIÓN: EL ESTADO DE DERECHO SUSTANTIVO}

Algunos autores, conscientes de los problemas que puede confrontar la noción de estado de derecho propuesta por Fuller para proteger derechos en democracia, han planteado la necesidad de superar aquella noción. Así, estos autores plantean que una correcta noción de estado de derecho no solo debería incluir dentro de sí las exigencias contenidas en los desiderata de Fuller, sino también otro tipo de exigencias materiales, como el respeto por la democracia, la justicia social, la autonomía individual o los derechos humanos ${ }^{71}$. En este contexto, podría, en efecto, parecer positivo adherir a un

${ }^{70}$ Un ejemplo de esto lo representa la serie de casos resueltos por la Corte Interamericana de Derechos Humanos en relación con los excesos del régimen chavista en Venezuela, que llevaron a la condena del Estado venezolano por numerosas violaciones de derechos fundamentales. La estrategia de promover este tipo de litigios internacionales fue tan exitosa que el ejecutivo del Estado finalmente decidió denunciar la Convención en 2012. Similar cosa ocurrió con Perú durante el gobierno de Fujimori, en 1999.

${ }^{71}$ Tamanaha, op. cit., p. 102. 
concepto de estado de derecho que aparentemente garantiza de forma más robusta los derechos fundamentales.

Sin embargo, adherir a esta noción de estado de derecho sustantivo no es necesariamente conveniente ni para el estado de derecho ni para los derechos fundamentales.

Primero, por razones de claridad analítica ${ }^{72}$. En efecto, toda filosofía política se enriquece en la medida que ella es capaz de asociar con suficiente precisión el contenido de diferentes tipos de demandas con ciertos términos técnicos, como estado de derecho, democracia, justicia o derechos fundamentales. Ahora bien, cada uno de estos términos encierra ciertas exigencias específicas que deben ser diferenciadas con el propósito de permitir un entendimiento más acabado del significado y alcance de esos términos. Asimismo, realizar esa diferenciación permite estudiar la forma cómo esos términos políticamente relevantes interactúan entre sí en la práctica. Desde esta perspectiva, mezclar distintos tipos de exigencias -que incluso podrían resultar ser contradictorias entre sí- bajo el epígrafe de estado de derecho resta transparencia conceptual a los mencionados análisis. Esto porque hace más difícil entender el alcance de la noción de estado de derecho -ya que impide su adecuada diferenciación de otros conceptos-, y limita la comprensión de su interacción con otros términos - en la medida que la aproximación criticada busca unificar forzosamente términos que expresan requerimientos de naturaleza diversa-.

La confusión conceptual acerca del alcance de la noción de estado de derecho sustantivo se torna aún más intensa en cuanto no existe consenso entre los autores respecto de qué criterios de legitimidad material incluir dentro del señalado concepto ${ }^{73}$. Desde esta perspectiva, la gran diversidad y equivocidad de las nuevas exigencias propuestas amenazan con vaciar de contenido la noción misma de estado de derecho, el que podría tener tantos significados como elementos pueda poseer ${ }^{74}$. En este escenario, la idea de estado de derecho pasaría a constituir un mero eslogan que permitiría justificar todo tipo de doctrinas iusfilosóficas acerca de la justicia y los derechos humanos ${ }^{75}$. Esto último también terminaría por oscurecer el valor y la relevancia de los desiderata planteados por Fuller, cuya aceptación es generalizada entre los autores que proponen la adopción de un modelo sustantivo de estado de derecho ${ }^{76}$.

Segundo, porque este modelo de estado de derecho sustantivo no asegura necesariamente mayores grados de protección a los derechos fundamentales en democracia que aquellos que puede ofrecer el modelo propuesto por Fuller. En este sentido, la reacción de las democracias ante la amenaza global del terrorismo es una muestra clara de que la adhesión de aquellas a un modelo de estado de derecho sustantivo no asegura necesariamente

${ }^{72}$ Raz, J., "The rule of law and its virtue", en Raz, J. (autor), The authority of law, Oxford University Press, Oxford, 2012, p. 211.

73 Bingham, op. cit., p. 86.

${ }^{74}$ Shklar, J., "Political theory and the rule of law", en Hutchenson, A. y Monahan, P. (edit.), The rule of law: ideal or ideology?, Carswell, Toronto, 1987, p. 1.

${ }^{75}$ Raz, op. cit., 2012, p. 211.

${ }^{76}$ E.g: Dworkin, R., "Political judges and the rule of law", en Dworkin, R. (autor), A matter of principle, Harvard University Press, Cambridge Massachusetts, 1985, pp. 11-12. 
la protección de derechos fundamentales. Por el contrario, pese a las demandas de aquel modelo de estado de derecho, las legislaturas democráticas de países como Estados Unidos han terminado, finalmente, cediendo a las exigencias planteadas por la seguridad nacional o el orden público, por ejemplo, en detrimento de los derechos fundamentales ${ }^{77}$.

\section{CONCLUSIÓN}

El presente trabajo ha buscado determinar si la noción de estado de derecho propuesta por Fuller tiene capacidad para proteger derechos fundamentales. Tras analizar la forma como opera la noción en cuestión tanto en el contexto de regímenes que persiguen derechos como de regímenes que los respetan, es posible afirmar que, efectivamente, aquel modelo de estado de derecho tiene capacidad para proteger derechos fundamentales. Esto, básicamente, porque las exigencias del estado de derecho limitan la capacidad de maniobra de toda autoridad que busca infringir derechos fundamentales -sea esta totalitaria, dictatorial o democrática- disminuyendo, de esa forma, su maligna eficacia.

\section{BIBLIOGRAFÍA}

Libros, revistas y artículos

Abel, R., "Legality without a Constitution: South Africa in the 1980s", en Dyzenhaus, D. (editor), Recrafting the rule of law, Hart Publishers, Oxford, 1999, pp. 66-80.

Bingham, T., The rule of law, Penguin Books, Londres, 2011, 224 pp.

Calabresi, G., "Two functions of formalism: in memory of Guido Tedeschi”, The University of Chicago Law Review, Vol. 67, 2000, pp. 479-488.

Douglas-Scott, S., Law after modernity, Hart Publishers, Oxford, 2013, 428 pp.

Dworkin, R., "Political judges and the rule of law", en Dworkin, R. (autor), A matter of principle, Harvard University Press, Cambridge Massachusetts, 1985, pp. 9-32.

FinNis, J., Natural law and natural rights, Oxford University Press, Oxford, 2011, 494 pp.

Fuller, L., The morality of law, Yale University Press, New Haven, 1969, 262 pp.

Fuller, L., "The implicit laws of lawmaking", en Winston, K. (edit.), The principles of social order. Selected essays of Lon L. Fuller, Hart, Oxford, 2001, pp. 175-186.

GEORGE, R., "Reason, freedom, and the rule of law: their significance in the natural law tradition”, American Journal of Jurisprudence, Vol. 46, 2001, pp. 249-256.

Grzybowski, K., "Main trends in the soviet reform of criminal law", The American University Law Review, Vol. $9 \mathrm{~N}^{\circ}$ 2, 1960, pp. 93-110.

Muller, I., Hitler's justice: the courts of the Third Reich, Harvard University Press, Cambridge Massachusetts, 1991, 349 pp.

Peerenboom, R., "Human rights and the rule of law: what's the relationship?", Georgetown Journal of International Law, Vol. 36, 2004-2005, pp. 809-945.

${ }^{77}$ E.g.: Waldron, J., "Torture and positive law: jurisprudence for the White House", Columbia Law Review, Vol. 105, 2005, pp. 1681-1750. 
Raz, J., "The politics of the rule of law", en Raz, J. (autor), Ethics in the public domain, Oxford University Press, Oxford, 1995, pp. 370-378.

RAz, J., "The rule of law and its virtue", en Raz, J. (autor), The authority of law, Oxford University Press, Oxford, 2012, pp. 210-229.

Rundle, K., Forms liberate. Reclaiming the jurisprudence of Lon L. Fuller, Hart Publishers, Oxford, 2013, 222 pp.

ShKlar, J., "Political theory and the rule of law", en Hutchenson, A. y Monahan, P. (edit.), The rule of law: ideal or ideology?, Carswell, Toronto, 1987, pp. 1-16.

Simmonds, N., Law as a moral idea, Oxford University Press, Oxford, 2007, 220 pp.

Simmonds, N., "Straightforwardly false: the collapse of Kramer's positivism", The Cambridge Law Journal, Vol. 63 No 1, 2004, pp. 98-131.

Summers, R., "A formal theory of the rule of law", Ratio Iuris, Vol. $6 \mathrm{~N}^{\circ}$ 2, 1993, pp. 127-142.

Tamanaha, B., On the rule of law. History, politics, theory, Cambridge University Press, Cambridge, 2004, 180 pp.

Thompson, E.P., Whigs and hunters, Penguin Books, Londres, 1977, 278 pp.

WALdron, J., "The rule of law in contemporary liberal theory", Ratio Iuris, Vol. 2 No 1, 1989, pp. 79-96.

Waldron, J., "Torture and positive law: jurisprudence for the White House", Columbia Law Review, Vol. 105, 2005, pp. 1.681-1750.

Waldron, J., The rule of law and the measure of property, Cambridge University Press, Cambridge, 2012, 133 pp.

Webber, G., The negotiable constitution. On the limitations of rights, Cambridge University Press, Cambridge, 2012, 231 pp.

\section{Sentencias del Tribunal Europeo de Derechos Humanos}

Caso Sunday Times vs. Reino Unido. Sentencia de 26 de abril de 1979.

Caso Huvig vs. Francia. Sentencia de 24 de abril de 1990.

Caso Herczegfalvy vs. Austria. Sentencia de 6 de noviembre de 1992.

Caso Steel y otros vs. Reino Unido. Sentencia de 23 de septiembre de 1998.

Caso Maestri vs. Italia. Sentencia de 17 de febrero de 2004.

Caso Gillan y Quinton vs. Reino Unido. Sentencia de 12 de enero de 2010.

\section{Sentencias de la Corte Interamericana de Derechos Humanos}

Caso Baena Ricardo y otros vs. Panamá. Sentencia de 2 de febrero de 2001.

Caso Fermín Ramírez vs. Guatemala. Sentencia de 20 de junio de 2005.

Caso Kimel vs. Argentina. Sentencia de 2 de mayo de 2008.

Caso Tristán Donoso vs. Panamá. Sentencia de 27 de enero de 2009.

Caso Torres Millacura y otros vs. Argentina. Sentencia de 26 de agosto de 2011. 
